\title{
Undergraduate education in radiology. A white paper by the European Society of Radiology
}

\author{
European Society of Radiology (ESR)
}

Received: 3 May 2011 / Accepted: 4 May 2011

(C) European Society of Radiology 2011

\section{Introduction}

by Éamann Breatnach, Chairman of the ESR Education Committee

A common goal of all initiatives undertaken by the ESR Education Committee is the improvement and transnational harmonisation of educational standards in radiology throughout Europe. High standards and uniformity of undergraduate education in radiology are essential components in this effort. Though the European Society of Radiology is not a regulatory authority, by providing references and guidance documents, it can beneficially influence educational authorities at both a national and local level.

The Executive Council of the ESR, by establishing a working group on undergraduate teaching reporting to the Education Committee, has formally endorsed the area of radiologic education to be a priority. The policy is in line with other major initiatives being undertaken by the Society, in particular revision of the Post Graduate Charter and Curriculum for Radiology Training (February 2011, www.myesr.org/trainingcharter) and the development of a Diploma in General Radiology (www.myesr.org/diploma) to be taken at the end of residency training.

Through the organs and structures of the Society including the Executive Office and all statutory committees of the Society, the ESR plans dissemination of this white paper throughout the European radiology community. By

European Society of Radiology (ESR) $(\bowtie)$

Neutorgasse 9/2,

AT-1010 Vienna, Austria

e-mail: communications@myESR.org including the white paper as an agenda item at all statutory committees within the ESR, the Society will promote involvement of radiologists in undergraduate programmes on as wide a basis as possible. It is also proposed that a specific focus be made on dissemination to the University authorities in Europe who have responsibility for medical student education. A database of these institutions has been created within the offices of the ESR. In addition, on occasions of consultation with national leaders, e.g. Leadership Meeting, the Education Committee will actively promote the subject of undergraduate education and this white paper to as wide an international audience of radiology leaders as possible. The Executive Office has also sanctioned proposals from the Undergraduate Teaching Working Group to elaborate supplemental promotional literature to be sent to institutions across Europe with responsibility for undergraduate education.

To promote further awareness, a proactive focus on undergraduate education will be proposed to the ECR Programme Planning Committee. This diffusion will be supplemented by a pro-active focus on undergraduate education at the ECR Programme Planning Committee. The public relations expertise and facilities of the Society and Congress will be available to support the concept of radiology as a career choice for our brightest students by actively encouraging attendance of school leavers and medical students at specific age-appropriate promotional events within the Congress e.g. the ESR Rising Stars - Residents and Students Programme (www.myesr.org/risingstars).

The Executive Council is aware of the hard work and enthusiasm that the Working Group in Undergraduate Teaching has brought to the subject and endorses fully the outlined content of the proposed white paper as suggested. 


\section{Executive summary}

The ESR has a justifiable role in promoting transnational advice on appropriate teaching of radiology to undergraduates, for which there appears to be significant need. This role is supported by a number of objectives apposite to the role of the ESR in European medical practice. This white paper encapsulates a survey of current (2010) undergraduate teaching in Europe, which indicates a promising base of activity on which the present advice can build. Images have particular strengths as teaching tools, and a wide range of teaching methods are appropriate to radiology. Recommendations are made on how to involve radiologists in undergraduate programmes, and how to expose undergraduates practically to radiology services. The ESR has an important role in encouraging, advising and supporting radiologists engaged in undergraduate teaching, and in acting as a channel for mutual support and communication amongst teachers. All European medical schools should ensure that a core curriculum of radiology is delivered to their undergraduates. A suitable framework for this core is specified.

\section{Contents overview}

1. Preamble

2. Objectives of undergraduate teaching in radiology

3. Current activity in Europe-findings of the 2010 ESR survey

4. Teaching methods appropriate to radiology-the possibilities

5. Involving the radiologist in the teaching programme

6. Engaging the undergraduate in the radiology service

7. Communicating with the teachers

8. A proposed curriculum framework

\section{Preamble}

In March 2010, the European Society of Radiology (ESR), through its Education Committee, established a Working Group on Undergraduate Teaching in Radiology, in pursuit of the Society's aims. The underlying rationale is that introducing undergraduates to radiology is concordant with the responsibilities of the ESR to the medical community and therefore the Society has a role in advising on this teaching activity.

It is accepted that circumstances in universities differ significantly across Europe and that it is not possible to prescribe a standardised approach to undergraduate education. The Working Group sets its report within the concept of a general framework of advice, which the ESR offers to European universities.
The Working Group regards the white paper as the initial step in a continuing process within the ESR and foresees further steps, some of which are recommended in the white paper and more of which will follow from discussion within the ESR Education Committee. The members of the Working Group are committed to supporting such further initiatives as the Executive Council decides.

\section{Objectives of undergraduate teaching in radiology}

The over-arching concern of the ESR is that effective practice of radiology should contribute to increasing standards of patient care. This objective operates throughout medical training and subsequent clinical practice and is a continuous process.

The involvement of radiologists in teaching medical undergraduates is a logical beginning to this process. By ensuring that undergraduates are taught radiology, several objectives important to them at this stage of their education and also to the practice of radiology itself may be served. These objectives consist of the following:

1. To ensure that undergraduates are equipped with the knowledge and interpretational skills they will require to practise safely and appropriately in their early career, providing a knowledge base of the principles of radiology on which to build as their career develops. This should comprise some familiarity with the following:

- Anatomy and physiology as pertaining to clinical radiology

- Imaging physics and radiation protection

- The characteristics of imaging techniques

- The clinical role of imaging techniques, both individually and as part of a coordinated investigation regime

- The use of appropriate referral criteria and clinical guidelines

- Appropriate investigation of acute and lifethreatening conditions

- Interpretative skills for emergency investigations

2. To ensure that medical graduates are fully aware of their legal responsibilities with regard to patient care and safety as influenced by radiology.

3. To provide an awareness of the importance of resource management in health care and costs and benefits of radiology in relation to clinical management.

4. To provide an awareness of developments in radiology that can be anticipated to form part of the clinical practice in their future careers. 
5. To support undergraduate learning across the rest of the clinical curriculum by exploiting the power of images to elucidate normal and pathological anatomy and the nature and behaviour of disease.

6. To raise the profile of radiology as a career choice for undergraduates.

\section{Current undergraduate teaching activity in European radiology—findings of the 2010 ESR survey}

The 2010 ESR survey is an extension of a previous survey carried out in 2008 [1] and addressed the questions:

1. How is undergraduate teaching delivered?

2. In what medical school year(s) do students study radiology and what proportion of the curriculum focuses on radiology or radiology topics?

3. What types of staff are responsible for radiology education?

4. What radiology topics are examined and how are they examined?

The present web-based questionnaire was electronically distributed by the ESR in July 2010 to radiology teaching staff and chiefs of teaching hospitals in 430 European teaching centres. Ninety-three responses $(21.6 \%)$ were obtained from 26 countries. Each country was represented by one or more teaching centres. A detailed analysis is being prepared for publication. The leading results presented here provide a panoramic view of how radiology teaching is currently delivered and organised in European educational centres.

The average duration of the preclinical phase of medical undergraduate education is 2.2 years. The average duration of the clinical phase is 3.7 years. The mean number of the students enrolled in the last year of medical school across all responding educational centres is 156.5 .

How is undergraduate teaching delivered?

Most of the centres $(92 \%)$ reported radiology courses as a part of formal curriculum.

The teaching approach used in undergraduate education differs across countries. In more than half of the centres, radiology is taught as an independent discipline with its own examination (the "classical" model). A hybrid approach (the combination of classic and modular type of teaching) is practiced in $32 \%$ of centres.

A "modular" type of teaching, where radiology is integrated within clinical thematic teaching modules (covering systems or parts of the body such as thorax, abdomen, musculoskeletal system, nervous system, and urogenital system) dominates in $20 \%$ of teaching centres. In this modular approach radiology is not presented as a separate discipline and is therefore relatively underrepresented in examinations (so that students may be tempted to "skip" radiology and radiological anatomy). In this approach, teaching on the nature of imaging techniques (explanation of radiography, CT, ultrasound, MRI, angiography, interventional radiology and contrast media) is usually incorporated in the pre-clinical part of the training.

e-learning for radiology is available in $74 \%$ of centres and is usually based on teaching files using the World Wide Web or PACS (or Web-based PACS) in more than $30 \%$ of centres. Teaching files on $\mathrm{CD}$, with or without specific education software developed by the institution itself, also appear popular (more than $20 \%$ of centres).

Sessions that provide undergraduates with direct experience of radiology services_- "practical sessions", "sign-off sessions" or "clerkships"- appear commonly (91\%) to be a key part of undergraduate radiology teaching, especially during the clinical part of the medical training program (third year $32 \%$, fourth year $53 \%$, fifth year $42 \%$ ). These clinical clerkships are obligatory in $47 \%$ of centres and elective in $44 \%$.

Information was gathered about tasks and responsibilities during the clerkships. The list of tasks and responsibilities returned included the following:

- Observing routine clinical practice in the department: patient flow, image flow, administrative and logistic work, etc., $76 \%$

- Following a selected number and type of radiological examinations, $70 \%$

- Attending clinicoradiological conferences, $60 \%$

- Attending multidisciplinary team meetings, $39 \%$

- Working with teaching files via PACS, $32 \%$, or on CD-ROM,19\%

In what medical school year(s) do students study radiology and what proportion of the curriculum focuses on radiology or radiology topics?

On average, the largest proportion of radiology is presented during the fourth year of training in most countries $(69 \%)$, followed by third and fifth year of training ( $53 \%$ for each). Only $20 \%$ of centres reported providing initial experience with radiology in the first year.

The number of curricular teaching hours focusing on radiology topics differs across centres. A relatively large proportion of teaching hours (average $40 \mathrm{~h}$ ) devoted to radiology of diseases is typical of most centres, followed by hands-on training, including interpretation skills (average $19.6 \mathrm{~h}$ ), radiological anatomy (average $17.5 \mathrm{~h}$ ) and radiological techniques (average $13.7 \mathrm{~h}$ ). The average number of teaching hours is less extensive for interven- 
tional radiology (average $5.3 \mathrm{~h}$ ), radiation protection (average $4.3 \mathrm{~h}$ ) and for guidelines for appropriate use of radiology (average $5.9 \mathrm{~h}$ ).

As noted above, the third, fourth and fifth years-the clinical phase - of medical training are currently reported to be the most important years for teaching radiology. Radiological topics, such as imaging of diseases, radiological techniques, radiation protection, interventional radiology, hands-on experience, interpretation skills, and guidelines for appropriate use of radiology, are mostly taught during the clinical phase of education, whereas radiological anatomy is usually taught during the first and second years.

What types of staff are responsible for radiology education?

Although from a didactic point of view restricting the number of teachers to one or two dedicated, academically oriented radiologists might be the ideal method for undergraduate teaching (by keeping the same structure and learning methods in the education process), current practice appears different. In most European training centres, radiology topics are taught by more than two staff members $(89 \%)$. The average number of radiology-related teaching staff is 13 , ranging from 1 dedicated teacher to 64 staff members or consultants involved in the whole teaching process.

Centres report that anatomy and radiological techniques are preferably taught by general $(75.8 \%)$ or subspecialised $(62.4 \%)$ radiologists. Radiology of disease is more preferably taught by subspecialised radiologists $(82.8 \%)$ as is, predictably, interventional radiology (87.1\%). Teaching in radiation protection involves physicists in $38.7 \%$ of departments; apart from these specialised areas, junior staff are involved in teaching in $18.3 \%$ of centres.

The advantage of involving subspecialised radiologists is that they are more familiar with radiological subspecialities and can teach with more confidence. However, the risk is that they may go too much into details that are beyond the learning objectives for undergraduate students.

What radiology topics are examined and how are they examined?

Radiology examinations appear mostly set up as a part of larger clinical examinations (the "modular" approach, together with other disciplines). Exceptions are separate examinations in relation to radiology techniques $(28 \%)$, interventional radiology $(20 \%)$, radiation protection $(29 \%)$ and hands-on examinations (13\%).

Written tests and/or oral examinations are the most commonly used methods for student evaluation (range 10-50.5\%). Computer-based evaluation and/or Objective
Structured Clinical Examination (OSCE) evaluation are also used but not widely (range $3.2-17.2 \%$ ).

\section{Teaching methods appropriate to radiology—-the possibilities}

\section{General principles}

Radiology teaching should be undertaken as part of an agreed curriculum and agreed programme of delivery. The programme should be published and available to all students and all teaching staff. The radiology curriculum should be integrated with the general curriculum to ensure that teaching of radiology is in harmony with students' learning objectives across the whole of their training.

Medical schools differ in their approach, and it is probable that individual schools or departments will wish to capitalise on particular strengths. This is desirable but should not be allowed to overbalance the teaching programme. There is a critical core of radiology that is needed by all students, and this must form the basis of the teaching programme. A "core plus options" approach may be used. A framework for core curriculum is given in the section "A proposed curriculum framework".

It is desirable to ensure a radiology presence across the whole curriculum where possible, from introductory subjects and basic sciences to revision before final examinations.

Students tend to be more receptive to teaching that they perceive to be relevant to their current needs and which supports their learning. Sensitivity is needed in the radiology teaching programme to students' learning needs at each stage of their degree course. This is well served by ensuring that the radiology programme is coordinated with the general curriculum. It is also strengthened when part of the radiology programme exploits the advantages of images in helping students' learning in other subjects such as anatomy, pathology and the natural history of disease.

The advantages of images as a teaching tool

Teaching programmes should exploit as far as possible the characteristics of images that make them powerful teaching tools. Images evoke powerful memory cues ("every picture tells a story"). They can be associated with clinical scenarios or key teaching points to aid students' understanding or retention of important subjects.

Images may be used to assist teaching in this way throughout the whole curriculum. Although there are learning outcomes which are specific to radiology, teaching radiologists should not neglect this important contribution 
to student experience and to the work of their medical school. Subjects to be considered are as follows:

- Anatomy

- General pathology

- Clinical effects of lesions

- Natural history of disease

- Responses to treatment and complications of therapy

Radiologists who follow this approach are likely to win greater recognition for the speciality within their medical school.

The radiology teaching programme should be structured so that students are not exposed to unfamiliar subjects without appropriate previous teaching.

Particular aspects of images will inevitably suit some subjects in the clinical curriculum more than others. It is important to adapt teaching methods to the individual learning objectives at each point in the course; a "horses for courses" approach will be most rewarding to both student and teacher.

The advent of digital imaging has facilitated the use of images in teaching. Particular advantages include the following:

- Creation of generic or shared teaching files

- Creation of standardised examination questions

- Incorporation into computer-assisted learning (CAL) packages and self-teaching exercises

- Three-dimensional display of anatomy and disease

- Virtual reality (VR) teaching and training programmes for clinical skills

PACS and Web-based PACS on personal computers that are exclusively available for students are essential for hands-on teaching and improvement of interpretation skills of students. Radiologists can create "teaching" maps on PACS workstations, where they collect routine, illustrative cases, avoiding complexity or rarity.

The teaching environment

Effective teaching programmes need accommodation that serves good learning. Radiologists should have access to rooms and technical facilities that allow effective teaching.

Where possible, student engagement with radiology should be encouraged by providing teaching within the department of radiology.

It is important that students also see radiologists engaged with clinical colleagues. The learning impact of allowing students to observe the interaction between radiologists and clinical referrers, for example in clinico-radiological review meetings, should be acknowledged.

It is probable that most radiology teaching will be delivered to small groups of students. Although it is not the only approach, this makes it possible for students to have close contact with the images being used, especially when display is by computer or workstation.

Although small group teaching is likely to predominate, large group teaching may be required at times. Examples include introductory and revision classes. An alternative, and appropriate resource if available, is the CAL classroom, where students have individual computer stations. These classrooms are becoming established in European medical schools, and radiologists should ensure that they have access to this facility.

\section{Teaching delivery}

All teachers have individual strengths, and choice of teaching delivery is likely to be influenced by personal preference. This may potentially be a strength in the programme. However departments should ensure wherever possible that students experience a pre-determined core knowledge and are exposed to a variety of teaching methods, and that teaching radiologists are prepared to exploit the relative merits of each of these. The methods outlined below are perceived to have particular advantages in teaching radiology.

\section{Problem-based learning (PBL)}

The basic principle of PBL is to provide students with a perceived problem and ask them to explore solutions in group discussion. PBL is based on self-learning by the student, and the tutor acts as discussion facilitator, not necessarily as content expert. Radiology is particularly suitable for PBL because images are an essential element of management in almost all disease processes, meaning that they can be readily integrated into clinical PBL scenarios ("every picture tells a story").

PBL was developed as a student-centred method based on "problems" in general, but these can be any problematic situation, case or scenario that produces discussion. Small group teaching is the norm and the students develop the learning strategy themselves. Full explanations of the method are available in specific reviews.

Advantages of PBL include the following:

- PBL students learn as well, if not better, than in traditional programmes and develop excellent group and cognitive analytical skills.

- PBL students show more motivation, commitment and constructive behaviour.

- Use of learning resources-books, journals, etc.-is stimulated.

- Students report high satisfaction with education system and teachers. 
- PBL allows the development of generic skills and team work, which is valuable in professional life.

- $\quad$ PBL is enjoyable for both the student and facilitator.

Limitations include the following:

- Final knowledge and competence are similar to traditional methods.

- If all teaching is delivered in this way, there is a risk regarding knowledge of basic science, which is more appropriately taught by traditional methods.

- Most medical schools have limited time to cover the basic knowledge of image interpretation and patient safety, and guidelines to direct use of PBL to appropriate areas are desirable.

- PBL is more time-intensive and therefore resourcedemanding than traditional methods. This may be alleviated by teaching in larger groups.

- PBL can be frustrating for tutors who find it difficult to guide discussion. Training and inter-tutor discussion of experiences may avoid this.

- The evidence base of benefit in radiology is limited. Schools should establish their own feedback systems to evaluate how PBL serves their students.

\section{Self-teaching packages}

These are growing in popularity among students as they allow private study on the time scale the student finds appropriate. This popularity is reflected in the number of self-teaching books on the market.

Self-teaching is best regarded as an addition to, and not substitute for, a core teaching programme. Departments are advised to develop their own teaching packages to support their particular teaching programme. This is ideally done in coordination with clinical referrers, to support the learning objectives defined by both groups.

Incorporation of images into computer-based teaching packages provides this delivery in an e-learning form.

\section{Handbooks, handouts, exercises}

Departments can assist student learning significantly by providing visual written material specific to their own teaching programme. Handbooks have a particular value in directing further study. It is good practice for students to receive handouts of all formal presentations before the event.

Exercises are valuable to improve knowledge and interpretation skills. Exercises on radiological anatomy can be found on the internet. Published exercises on radiological aspects of disease frequently consist of rare or unusual cases, so dedicated packages are required. Exercises may be based on different types of questions such as multiple choice, fill-in-the-blank(s), open questions, and "click on" answers in e-learning packages. Learning reinforcement is served if exercises provide immediate feedback to correct errors and emphasise correct answers.

\section{Guided case learning}

A new didactical teaching method is "guided case-based learning", where radiologists who are performing their routine daily clinical work select a few cases from their work list on PACS and ask the students to study these cases independently or in groups with other students, via Web-based PACS on personal computers in the department exclusively reserved for student study. Thereafter, students can return and discuss their findings and conclusions with the radiologist.

\section{Use of the internet or hospital or department intranet}

Facilities for easy access by students to the internet should be available within the teaching department. Students become progressively attuned to finding information electronically and departments should exploit e-learning wherever possible. Internet facilities are particularly applicable to self-teaching packages, which students may access at times and places that suit their needs. Internet communication is also a suitable medium for conveying guidelines on the appropriate use of imaging techniques, and on safety considerations.

Use of the internet holds significant possibilities for shared teaching material, such as Web-based teaching archives for use by teachers, self-teaching packages for the use by students and self-assessment or examination packages. There is a role for international societies in producing such packages.

\section{Involving the radiologist in the teaching programme}

Radiology is an important part of the clinical curriculum, and teaching is therefore one of the primary functions of a department of radiology. Departments must make staff available for teaching and must provide appropriate supporting facilities.

Teaching should extend throughout the curriculum, and radiologists must have a profile which is visible to undergraduates. Radiologists should ideally be involved at the outset of the clinical course, for example by taking part in introductory or basic science teaching.

Departments must bid for teaching time in competition with the claims of other departments in the curriculum.

Factors dictating choice of which radiologist should teach include the subspeciality experience and level of 
interest of the individual, and their competing duties. To satisfy the curricular objectives departments will probably need to call on the services of most, if not all, departmental staff at some time, though few individuals are likely to be required throughout the whole course.

Departments should provide protected time both for teaching and for teaching preparation. They should also provide adequate stimulation and recognition of teaching in order to maintain workforce commitment. The section below outlines some approaches that are relevant.

Increasing recognition of teaching possibilities

The member who leads undergraduate teaching should communicate clearly the academic vision for radiology teaching, the learning objectives and the education mission. The radiology curriculum should be presented and explained to the staff, so that subspecialist radiologists can be involved in teaching separate topics according to educational need.

The interest or expertise in teaching of individual radiologists may vary within a department, and there should be access to appropriate guidance for radiologists who wish to increase their skills. Access to the teaching literature is important, as is provision for radiologists to attend training courses on teaching.

Through discussion, departments should ensure that each member of the staff has personal teaching tasks and responsibilities appropriate to their teaching capacities and availability. Tasks may range from daily support during radiological clerkships to more didactical presentations. Teaching radiologists should be encouraged to obtain feedback from their students to evaluate their teaching and this should be monitored by departments, preferably annually. Heads of departments should ensure that teaching within their remit carries recognition and consider rewarding outstanding commitment to teaching methods or development.

Advice on leadership, resource management, methods and equipment

One of the key hindrances of involving radiologists in teaching programmes is the high workload of radiologists in teaching hospitals. They are frequently overburdened with a variety of responsibilities, consuming much time and attention, and sometimes facing serious under-staffing. Therefore it is important to protect time for teaching, so that as many members of the department as possible can contribute to teaching and mentoring students.

To provide effective teaching, radiologists need a large number of images to illustrate courses and to improve the interpretation skills of students. Therefore it is important to involve most or all department radiologists in collecting images for teaching. A shared departmental image archive is advantageous. Image display equipment may also be a shared resource. It is important that departments take steps to ensure that they are equipped to an appropriate level to provide effective teaching.

Effective teaching programmes require leadership. In radiology departments, this should be provided by a small number of radiologists, generally no more than one or two. These should have sufficient time allocated to them to undertake this role effectively. Concentrating responsibility in this way provides uniformity of approach and defines the character of the radiology programme. These members should be responsible for coordinating the curriculum objectives and delivery and ensuring that other radiologists teach at a level and with delivery appropriate to their point in the teaching programme. Coordinating radiologists should ensure that all teaching staff in radiology have regular opportunity to share their views and provide departmental feedback.

The department chairperson should ensure that the teaching resource offered by their staff in training is of high priority. Junior radiologists are frequently enthusiastic about teaching. Teaching enhances their own learning and develops their transferable skills. Junior radiologists should be subject to the same curricular control and supervision as applies in the department's programme and should receive appropriate guidance and support. It is unlikely they will have acquired sufficient teaching material at an early stage of their careers and they require access to departmental image archives.

Establishing networks for intellectual exchange in teaching

Teaching is improved when the staff has the opportunity to compare their teaching practice with that of colleagues, either in the host institution or between centres. In radiology, the most accessible liaison is with teachers in other clinical departments and is likely to be served by ensuring that the radiology programme is integrated with the rest of the clinical curriculum through discussion.

Workload pressures on academic departments tend to limit time for interprofessional exchanges, but departments should consider establishing formal networks for mutual support, training and sharing of teaching approaches.

Teaching radiologists should be jointly involved in periodic educational meetings, teaching seminars, short courses and educational workshops for students. This may be combined with involvement in the residency programme where practicable.

National societies and colleges should actively facilitate such networking by incorporating programmes of 
undergraduate radiology education at annual congresses, scientific gatherings, etc.

\section{Recognition of excellence in teaching}

Teaching in radiology departments is a year-on-year activity that requires continuing input and renewal of content, usually in the face of competing calls on the radiologist's time and energy. Recognition of the contribution of staff to teaching is essential to maintain motivation and commitment.

The most important recognition occurs at the local level. Heads of departments and radiologists coordinating teaching should consider the following:

- Evaluate the effect of the teaching efforts and discuss the reasons for satisfaction or dissatisfaction regarding teaching activities and educational formats

- Provide feedback for staff members to ensure that the academic teaching objectives are achieved

- Recognise and reward staff who consistently demonstrate commitment to teaching (e.g. by teaching awards, academic titles, financial bonuses)

- Continue to report and monitor benchmarking educational data on a systematic basis:

- To ensure the maintenance of didactic teaching

- To develop appropriate strategies to enhance effective teaching pedagogy

- To stimulate reflective practices

- To maintain an appropriate teaching curriculum

This recognition should also occur within the overall undergraduate medical faculty structures, where representation by radiology should be integrated in all significant medical education strategic developments.

Recognition is also desirable at national and international level where appropriate. Societies such as the ESR have a potential role in recognising the contribution of outstanding teachers by providing recognised teacher status or awards.

\section{Engaging the undergraduate in radiology service}

General approaches

Those in charge of courses should ensure that radiology has a visible presence to undergraduates throughout their clinical course. This is conveniently achieved by integrating the radiology teaching programme with the rest of the curriculum. It is important to provide a programme that engages students' interest. This can be strengthened by making sure the subjects taught in radiology coincide with the learning requirements of the students at each stage of their clinical course. This "phasing" is described below.
Students should see that radiologists are enthusiastic about their subject. This should not tempt the radiologist to teach at a level or a complexity that is inappropriate for the undergraduate. Engagement of the student is improved when teaching is held within the department of radiology. It is important to make students feel welcome in the department. This may require appropriate management of facilities.

Teaching radiologists should remember that medical undergraduates are adults who have views about their own learning methods. Teaching programmes should be adapted by student feedback to ensure that delivery is in a form appreciated by the students. Departments should have in place arrangements for obtaining feedback from students on a regular basis.

The importance of phasing

When radiology is taught throughout the curriculum, it is important to correlate the subjects taught by radiologists with clinical subjects that students are studying at the time. This reinforces their learning. The content of radiological teaching is therefore influenced by this principle, but tutors should also give consideration to how phasing of teaching influences the method of appropriate delivery.

For example, the following approach might be suitable for preclinical years:

\section{Content: radiological anatomy and imaging techniques}

- Include techniques that show anatomy well (radiography, CT, MRI, angiography)

- Present all anatomic regions

- CAL teaching is valuable for computer-based assessment and examinations, asking students to identify structures

- Teaching may be provided in large groups

- Individual computer stations may be needed for selfteaching and assessment

Whereas the following might be appropriate to clinical years:

Content: radiology of diseases and appropriate use of radiology services

- Teach what should be requested at what times

- Learn interpretation of most frequent diseases

- Avoid exotic diseases and emerging techniques (confusing for basic doctors)

- Include interpretation of common diseases in assessment

- Correlate with clinical teaching blocks (e.g. thoracic diseases)

- Teaching is likely to be in small groups 
- CAL assessment is valuable and may require separate stations

- Assessment may be based on multi-choice computer options

- Knowledge of international or where available national clinical guideline recommendations

\section{Visits and clerkships}

In addition to providing teaching sessions in the radiology department, tutors should recognise the value of longer student visits to the department. These may serve different objectives, according to length and exposure. The following is a suggested model.

\section{Observational visits or structured-guided visits}

- Demonstrate equipment, patient flow, image flow, reporting, administrative and logistic work, etc.

- These visits are most valuable when students are considering management of disease.

Longer visits (e.g. 1 week)

- Provide specific and active tasks for student engagement.

- Students may be assigned to a particular resident or radiologist.

- Students may follow a number of radiological examinations and interventional procedures.

- Offer selected cases (which may be web-based or CAL) to develop interpretational skills; providing feedback to the student is required in this process.

- Offer specific tasks, for example, ask students to look for interesting cases and present them.

- Should preferentially be done late in the clinical course.

\section{Extended stays in radiology (e.g. several weeks or months)}

- Interest in radiology can be stimulated by allocating extended tasks such as clinical audit projects or involving students in ongoing research projects.

- Students may be attached to a particular radiologist and share their work programme. This approach is especially suitable for students who are or may become interested in radiology as a career.

All student visits, especially extended ones, create additional workload for radiologists and this must be provided for in department scheduling. The choice of type of visit is often dictated by the number of students it is possible to accommodate together in the department; extended visits are usually only possible as a course option open to a few students.
Departments should ensure that their arrangements for student visitors comply with all legal restraints regarding the presence in departmental work of personnel who are not yet medically qualified.

The role of assessment

It is a fundamental principle of education that students adapt their learning to the assessments they must satisfy. This applies both to formative assessment, which is taken during their course in order to guide their progress, and to summative assessment as occurs to evaluate their performance in particular periods or modules of their course, or for award of their degree. Radiologists should therefore ensure that they are involved in the assessment programmes of their medical school. This will ensure students' motivation to pursue learning in radiology.

It is important that radiologists take part in medical school assessment programmes such OSCE stations. However it is also desirable for there to be separate assessment in radiology as otherwise student learning objectives will be dominated by the other subjects in which they know they have to undergo assessment.

Student learning is stimulated by providing assessment within the department. CAL programmes are a resourceeffective means of achieving this, although mentoring by a radiologist complements the value of student self-assessment. It is important that feedback is provided to the student after each assessment.

\section{Recognition of student engagement}

Student learning is further enhanced by ensuring that highachieving and committed students receive recognition. This provides positive reinforcement for those students and encouragement for their peers.

Recognition may be at department, school or higher level. Possibilities include the following:

- Providing prizes for good performance

- Stimulating student presentations

- Involving students in local, national or international meetings

- Encouraging and supporting student publications

International societies such as the ESR have a role in providing recognition of those students who are likely to choose radiology as a career or to be high-flying members of the profession.

\section{Communicating with the teachers}

The underlying rationale of this white paper is that the objective of the ESR in promoting the practice of 
radiology across Europe is served in part by extending and developing the quality of radiology teaching to medical undergraduates.

It is accepted that circumstances will differ in different medical schools and that these schools will have their own local requirements for radiologists who wish to teach undergraduates. However the needs of teaching in the specialty are best served by communicating uniformity of purpose to those radiologists engaged in teaching undergraduates.

Some countries have established fora or societies for medical teachers, and in some cases there are societies or sections of societies specifically for teachers in radiology. Such organisations have profound advantages in allowing teachers to share ideas, approaches and new developments, and are to be encouraged.

International societies such as the ESR have a potentially important role in bringing together teachers in radiology to ensure uniformity of purpose. A formal or informal association of undergraduate teachers within the ESR would be very likely to encourage further interest in teaching and provide stimulation and guidance to those who already teach.

Other ways in which the ESR could help develop undergraduate teaching of radiology in Europe would be to publish guidance in the field in the form of circulars or training CD-ROMs and to monitor teaching activity and development by obtaining feedback from medical schools. This is currently represented by the 2008 and 2010 surveys described above.

\section{A proposed curriculum framework}

Each medical school should adhere as closely as possible to a standard core curriculum for radiology, as described in detail below. This core may be supplemented with additional features as the individual school finds practicable and desirable.

Each medical school should have a defined framework for undergraduate learning in radiology, and radiologists should be involved in creating the framework.

Teaching in radiology should be based on clinical subspecialties and involve all imaging modalities. The objective should be to learn radiology in the context of clinical management of disease.

The core curriculum for radiology should relate as closely as possible to the individual school's general curriculum.

Decisions about methods of delivery should take particular note of two major objectives. Firstly, teaching should support undergraduates' learning of clinical subjects throughout their clinical curriculum. Secondly, teaching in radiology should equip undergraduates to undertake medical practice in the early years of their clinical careers.

Three particular categories of learning apply to the clinical objectives of newly qualified doctors:

1. Having the appropriate attitudes to support acceptable clinical practice

2. Understanding the legal requirements of practice relating to radiology

3 Having the clinical knowledge and skills appropriate to clinical practice in the early years of their career

Appropriate attitudes

Newly qualified doctors need to demonstrate an understanding of the experience of patients undergoing investigation in the radiology department. They need the ability to relate this to communication with the patient in the clinical environment and to appropriate psychological preparation of the patient before investigation. Doctors need to understand the importance of effective communication and liaison between clinicians and radiologists, in both directions. These objectives are well served by ensuring that undergraduates are exposed to and involved in the work of the radiology department.

Legislation

Students need to acquire a working understanding of the following:

\section{Protection of the patient}

- The hazards of radiation and the concepts of cumulative exposure and differential radiosensitivity of tissues

- The value of alternative investigation without the use of radiation

- Awareness of which investigations deliver a large absorbed radiation dose

- Current regulations, including principles of justification and optimisation (EC 97/43)

- Recognition of increased risk during pregnancy and in children and of measures to avoid inadvertent irradiation in early pregnancy

\section{Informed consent}

- Understanding of the principle of informed consent and its relation to invasive investigation in clinical imaging and interventional radiology

Appropriate knowledge and skills base

Radiology is best taught as a "continuous thread" throughout the medical curriculum, so that learning includes basic 
sciences such as anatomy and pathology and extends to therapy and the clinical management of disease. The learning outcome is characterised by an understanding of the role of individual imaging techniques in the management of patients, including their strengths, limitations and risks. Included is knowledge of the preparation required for individual procedures, and of the principles of referral based on clinical history and clinical examination.

Students require understanding of the concept of structured investigation regimes and of the disadvantages of unjustifiable over-investigation. This includes the relationship of clinical imaging techniques to other investigations (for example endoscopy). Students will understand that appropriate referral for radiological investigation is justified by a supposition of change in clinical management.

Students should be taught the use of local and national or international guidelines as part of this programme.

Students should also demonstrate a basic knowledge of the clinical possibilities of image-guided procedures and minimally invasive treatment.

Adequate understanding and application of the subjects listed below requires students to have a core knowledge of the aspects of anatomy and physiology which pertain to radiological interpretation.

\section{Interpretation of basic radiological studies}

The learning outcomes are characterised by an ability to detect abnormalities on chest, abdominal and skeletal radiographs and relate the findings to clinical management. Students should also display a systematic approach to comprehensive interpretation of radiographs.

On completion of the programme the student should demonstrate knowledge and recognition of the subjects listed below.

\section{The chest radiograph}

- Cardiac enlargement

- Cardiac failure and pulmonary oedema

- Pleural effusion

- Pulmonary collapse and consolidation

- Misplaced endoluminal tubes and catheters

- Pneumothorax, including tension

- Pneumomediastinum and subcutaneous emphysema

- Hyperinflation of lungs

- Free gas beneath the diaphragm

- Detection of pulmonary and mediastinal masses

- Signs of acute vascular dissection, including trauma

\section{The abdomen}

- Small and large bowel obstruction

- Toxic megacolon
- Signs of intestinal perforation

- Urinary calculi

- Foreign bodies

\section{Skeletal radiographs}

- Common fractures in the limbs

- Fracture of femoral neck

- Fractures of the wrist and scaphoid

- Fractures of the shoulder, including dislocation

- Pelvic fractures

- Skull fracture

- Vertebral fracture, dislocation and stability

- Signs of osteoarthrosis and inflammatory joint disease

- Sclerotic and lytic metastases

Skills will include the ability to relate the mode of injury to the type and site of fracture; to classify simple, comminuted and compound fractures; and to understand the value of radiographic projections at right angles.

\section{Radiology in the management of disease}

Chest and cardiovascular disease

- Chest and cardiovascular emergencies

- Airways disease: asthma and obstructive disease

- Aneurysms and vascular dissection

- Pleural effusion

- Pneumonia and other infections

- Embolic disease

- Pulmonary neoplasms

- Haemoptysis

Gastrointestinal disease

- Abdominal pain

- Abdominal masses

- Abdominal trauma

- Inflammatory bowel disease

- Jaundice

- Hepatic neoplasms

Renal and urological disease

- Renal failure and urinary obstruction

- Haematuria

- Urological neoplasms

- Renal and urinary infection

Endocrine and breast disease

- Thyroid dysfunction and thyroid masses

- Adrenal masses and dysfunction

- Breast masses 
Oncological and marrow disease

- Principles of oncological staging by imaging and knowledge of common staging regimes

- Investigation of haematological disease including anaemia and leukaemia

- Basic knowledge of potential complications of oncological treatment and means of detection

\section{Musculoskeletal disease}

- Bone and soft tissue infection

- Bone and soft tissue trauma

- Investigation of disease of joints

- Diagnosis of undisplaced or stress fractures

- Investigation of spinal injury

- Investigation of neck and back pain

Neurological disease

- Head injury

- Imaging strategy in stroke

- Intracranial haemorrhage

- Spinal cord compression

- Intracranial neoplasms

Disease of the ear, nose and throat

- Inflammatory disease of face and neck

- Deafness

- Disease of paranasal sinuses
- Neoplasms of face, neck and thyroid gland

- Salivary disease

Diseases of childhood

- The principles of imaging in children, including protection of the patient and confidentiality

- Disease of the chest, gastrointestinal and genitourinary tracts in childhood

- Acute musculoskeletal lesions

Obstetric and gynaecological disease

- Investigation of suspected or abnormal pregnancy

- Post-menopausal bleeding

- Gynaecological neoplasms

- Investigation of pelvic pain

Acknowledgements This paper was kindly prepared by the ESR Working Group on Undergraduate Education (chairperson: S.J. Golding; members: J. D. Aquerreta Beola, É. Breatnach, M. Knox, D. Malone, Z. Morvay, S. Pedraza, E. Szabó, K. Verstraete). It was approved by the ESR Executive Council in March 2011.

\section{References}

1. Kourdioukova EV, Valcke M, Derese A, Verstraete KL (2010) Analysis of radiology education in undergraduate medical doctors training in Europe. Eur J Radiol. doi:10.1016/j.ejrad.2010.08.026. 